\title{
Gravitational field of a pit and maximal mass defects
}

\author{
José P. S. Lemos \\ Centro de Astrofísica e Gravitação - CENTRA, Departamento de Física, \\ Instituto Superior Técnico - IST, Universidade de Lisboa - UL, \\ Avenida Rovisco Pais 1, Lisboa 1049-001, Portugal, email: joselemos@ist.utl.pt
}

O. B. Zaslavskii

Department of Physics and Technology, Kharkov V. N. Karazin National University, 4 Svoboda Square, Kharkov 61022, Ukraine and Institute of Mathematics and Mechanics, Kazan Federal University, 18 Kremlyovskaya Street, Kazan 420008, Russia, email: zaslav@ukr.net

\begin{abstract}
A general relativistic solution, composed of a Zel'dovich-Letelier interior made of radial strings matched through a spherical thin shell at radius $r_{0}$ to an exterior Schwarzschild solution with mass $m$, is presented. It is the Zel'dovich-Letelier-Schwarzschild star. When the radius $r_{0}$ of the star is shrunk to its own gravitational radius $2 m, r_{0}=2 m$, the solutions that appear have very interesting properties. There are solutions with $m=0$ and $r_{0}=0$ that further obey $\frac{2 m}{r_{0}}=1$. These solutions have a horizon, but they are not exactly black holes, they are quasiblack holes, though atypical ones. Moreover, the proper mass $m_{p}$ of the interior is nonzero and made of one string. Hence, a Minkowski exterior space hides an interior with matter in a pit. These are the pit solutions. These pits thus show a maximal mass defect. There are two classes of pit solutions, the first encloses a finite string and the second a semi-infinite one. So these pits are really string pits, which can be seen as Wheeler bags of gold, albeit totally squashed bags. There is also another class, which is a compact stringy star at the $\frac{2 m}{r_{0}}=1$ limit with $m$, and thus $r_{0}$, nonzero. It is a typical quasiblack hole and it also shows maximal mass defect. A generic analysis is presented that shows that pit solutions with $\frac{2 m}{r_{0}}=1$ and $m=0$ can exist displaying maximal mass defects. The Zel'dovichLetelier-Schwarzschild star at the $r_{0}=2 \mathrm{~m}$ limit is actually an instance of the generic case. Notably, these three classes of static solutions yield the same spectrum of solutions that appear in critical gravitational collapse, namely, there are solutions that yield naked null singularities, which here are the two string pit classes of solutions, there are solutions that yield black holes, which here are represented by the class of compact stringy stars at the quasiblack hole limit, and the solutions that disperse away in critical gravitational collapse here are the static Zel'dovich-Letelier-Schwarzschild stars themselves. A thermodynamic treatment of the string pit and stringy star quasiblack hole solutions is provided, and connections to other solutions are mentioned.
\end{abstract}

\section{INTRODUCTION}

There is the question as to whether, in general relativity, there are static configurations with some matter interior solution coupled to an exterior vacuum solution, for which three conditions are satisfied altogether: The configuration has zero radius $r_{0}, r_{0}=0$, it also has zero spacetime, or ADM, mass $m, m=0$, and, notwithstanding, $r_{0}$ and $m$ obey $\frac{2 m}{r_{0}}$ finite. We will answer this question in the positive. To do so we analyze a generic case, a generic star composed, say, of a generic interior spherical symmetric static configuration with a Schwarzschild exterior. Then, as a nontrivial example, we specifically develop and present the Zel'dovich-Letelier-Schwarzschild star, which is a solution that matches the Zel'dovich-Letelier interior made of spherically symmetric dust strings up to some junction radius $r_{0}$ to the exterior Schwarzschild solution. We send $r_{0}$ to the gravitational radius of the star $2 m, r_{0} \rightarrow 2 m$, i.e., we take the highest compact star limit, or the quasiblack hole limit, of the configuration. Solutions with $m=0, r_{0}=0$, and $\frac{2 m}{r_{0}}=1$, and thus $\frac{2 m}{r_{0}}$ finite, do indeed appear. They also have a nonzero positive proper mass $m_{p}$. Thus, clearly these solutions have maximal mass defects. Since $m=0$, the exterior spacetime is Minkowski. But since $m_{p}$ is not zero, such solutions enclose some matter in one form or another in a spacetime pit, and are thus pit solutions. These pits are atypical quasiblack holes. In the Zel'dovich-Letelier-Schwarzschild limiting star there are two classes of pit solutions: The first encloses a finite string and the second a semi-infinite one. Hence, in these classes the pits can be described as string pits. These string pits resemble Wheeler bags of gold but are totally squashed. There is also another class in the Zel'dovich-Letelier-Schwarzschild limiting star, which is a compact stringy star at the $\frac{2 m}{r_{0}}=1$ limit with $m$ finite, i.e., a usual quasiblack hole. These solutions can have a thermodynamic treatment and have interesting connections to other solutions.

Some comments are necessary. (i) The Zel'dovichLetelier solution was discussed as an interior general relativistic solution by Zel'dovich [1] in connection with compact stars, and by Letelier [2] as clouds of strings. It then appeared in the context of global monopoles [3] and of string hedgehogs and vacuum bubbles [4]. One of the properties of the solutions is that the mass function $m(r)$ obeys $\frac{2 m(r)}{r}=b$, for some fixed $b$ with $b \leq 1$. Zel'dovich 1] discussed the example of stars that are about to collapse and so the radial pressure is irrelevant, whereas Letelier [2] uses energy density $\rho$ and radial pressure $p_{r}$, but zero tangential pressure, with equation of state $p_{r}=-\rho$, which is also the equation used in [3, 4]. This means that one can envisage the matter source as string units emerging from a common center, and for this reason the source is called string dust, with the Zel'dovich setup being a particular situation of string dust, i.e., pure 
dust, since $p_{r}=0$. In brief, string dust has three features, namely, radial strings, $p_{r}=-\rho$, and no tangential pressure. We are interested in string dust solutions as interior solutions. We make use of the junction formalism [5] to match a Zel'dovich-Letelier interior to a Schwarzschild exterior and obtain the Zel'dovich-Letelier-Schwarzschild star. (ii) Objects for which the surface radius $r_{0}$ of the matter, e.g., a star, is at its own gravitational radius, $r_{0}=2 m$, are the highest compact stars called quasiblack holes [6]. Quasiblack holes are on the verge of becoming black holes, have special properties, and are relatives to both black holes and null naked singularities [6 -10]. (iii) The answer to the question as to whether, in general relativity, zero mass $m$ at a point $r=0$ can nonetheless have a quotient $\frac{2 m}{r}$ finite is known to be yes in a dynamical setting. Indeed, the inhomogeneous dust spherical collapse of the Lemaître-Tolman-Bondi models can produce an $m=0, r=0$, naked null singularity. At the critical moment the density has a $\frac{1}{r^{2}}$ isothermal profile, and $\frac{2 m(r)}{r}$ is finite, indeed $\frac{2 m(r)}{r}=1$ at the center [1113]. Also, Choptuik collapse of a scalar field [14] allows for a critical case, which divides expansion of the scalar field back to infinity from collapse of the scalar field to a black hole, where a zero mass and zero radius black hole, with $\frac{2 m}{r}=1$, and thus $\frac{2 m}{r}$ finite, forms. This can also be interpreted as the formation of a naked null zero mass singularity. (iv) Mass defects with maximal values have appeared in specific nonstationary models [15] where one can have matter with an infinite amount of interior proper mass, but the exterior spacetime has zero spacetime mass [16]. This is the maximal possible gravitational mass defect. (v) Bag of gold solutions appeared in [17], see also [18]. The Wheeler bags of gold are exemplified by a closed FLRW universe glued to the other side of a Schwarzschild black hole through an EinsteinRosen bridge with a knot at the junction closing the bag. (vi) Since the string pit and stringy star compact solutions found are quasiblack holes, it is of interest to discuss their thermodynamics as in [19, 20]. (vii) There are several related interesting solutions to the Zel'dovichLetelier interior that have the mass function $m(r)$ obeying $\frac{2 m(r)}{r}=b$ but are not string dust, i.e., the equation of state differs from $p_{r}=-\rho$, see, e.g., 21 28]. A match to a vacuum exterior of these related solutions does not yield Zel'dovich-Letelier-Schwarzschild stars.

The paper is organized as follows. In Sec. III we lay down the spacetime's basic features and arrive naturally at the concept of spacetime pits, i.e., solutions with $m=$ 0 and $\frac{2 m}{r}$ finite which yield maximal mass defects. In Sec. III we display the Zel'dovich-Letelier solution and make a proper matching to find the Zel'dovich-LetelierSchwarzschild star. In Sec.IV we take the quasiblack hole limit $r_{0} \rightarrow 2 m$ of the Zel'dovich-Letelier-Schwarzschild star and find three classes of objects with the highest compactification. The first two classes are pit solutions, more precisely, string pit solutions, one enclosing a finite string, and the other a semi-infinite string, both with $m=0, \frac{2 m}{r_{0}}=1$, and maximal mass defects. The third class is a compact stringy star, more precisely, a string star with highest compactness, i.e., $\frac{2 m}{r}=1$, finite $m$, and also a maximal mass defect. In Sec. $\left[\frac{r}{w}\right.$ we conclude, giving a synopsis with the results in a table, glimpsing through the thermodynamics of the string pit and stringy star solutions, and connecting with related work by others.

\section{BASIC FEATURES OF SPACETIMES WITH A PIT AND MAXIMAL MASS DEFECT}

\section{A. Spacetime generics}

A general static spherical symmetric spacetime with spacetime coordinates $(t, r, \theta, \phi)$ has a line element that can be written in the form $d s^{2}=$ $-\left(1-\frac{2 m(r)}{r}\right) \mathrm{e}^{2 \psi(r)} d t^{2}+\frac{d r^{2}}{1-\frac{2 m(r)}{r}}+r^{2} d \Omega^{2}$, where $m(r)$ and $\psi(r)$ are functions of $r$, and $d \Omega^{2}$ is the line element on the unit sphere, $d \Omega^{2}=d \theta^{2}+\sin ^{2} \theta d \phi^{2}$, and $\theta$ and $\phi$ are the angles on it. Assume that for $r \leq r_{0}$, for some radius $r_{0}$, there is a fluid with energy-momentum tensor $T^{a b}$ given by $T^{a}{ }_{b}=\operatorname{diag}\left(-\rho, p_{r}, p_{t}, p_{t}\right)$, where $\rho$ is the fluid's energy density, $p_{r}$ its radial pressure, and $p_{t}$ its tangential pressure, all functions of $r$. Then the Einstein equation of general relativity $G_{a b}=8 \pi T_{a b}$, where $G_{a b}$ is the Einstein tensor, and we put the constant of gravitation and the velocity of light to unity, yields $m(r)=4 \pi \int_{0}^{r} d r^{\prime} r^{\prime 2} \rho\left(r^{\prime}\right)$ and $\psi(r)=4 \pi \int_{r_{0}}^{r} d r^{\prime} r^{\prime} \frac{\rho\left(r^{\prime}\right)+p_{r}\left(r^{\prime}\right)}{1-\frac{2 m(r)}{r \prime}}$. There is yet another equation involving the tangential pressure $p_{t}$ that we do not need right now. In the model that we are going to use for the interior one has $\rho(r)+p_{r}(r)=0$ so that $\psi(r)=0$ throughout. Thus, in this case the line element that we start with in the $(t, r, \theta, \phi)$ coordinates reduces to

$$
d s^{2}=-\left(1-\frac{2 m(r)}{r}\right) d t^{2}+\frac{d r^{2}}{1-\frac{2 m(r)}{r}}+r^{2} d \Omega^{2},
$$

where

$$
m(r)=4 \pi \int_{0}^{r} d r^{\prime}{r^{\prime}}^{2} \rho\left(r^{\prime}\right)
$$

is now the only metric function, usually called the mass function and defined for $r \leq r_{0}$.

Other functions of interest here are the proper mass $m_{p}(r)$, the proper distance $l_{p}(r)$ from the center to any $r \leq r_{0}$, the area $A(r)$ of a constant $r$ sphere, and the proper volume $V_{p}(r)$. The proper mass $m_{p}(r)$ is defined as

$$
m_{p}(r)=4 \pi \int_{0}^{r} d r^{\prime} \frac{r^{\prime 2} \rho\left(r^{\prime}\right)}{\sqrt{1-\frac{2 m\left(r^{\prime}\right)}{r^{\prime}}}},
$$

the proper distance $l_{p}(r)$ from the center to any $r$ is defined as

$$
l_{p}(r)=\int_{0}^{r} \frac{d r^{\prime}}{\sqrt{1-\frac{2 m\left(r^{\prime}\right)}{r^{\prime}}}}
$$

the area $A(r)$ of a constant $r$ sphere is

$$
A(r)=4 \pi r^{2}
$$


and the proper volume is defined by

$$
V_{p}(r)=4 \pi \int_{0}^{r} d r^{\prime} \frac{{r^{\prime}}^{2}}{\sqrt{1-\frac{2 m\left(r^{\prime}\right)}{r^{\prime}}}} .
$$

These functions at the boundary $r_{0}$ become specific important quantities and we put

$$
\begin{array}{ll}
m \equiv m\left(r_{0}\right), & m_{p} \equiv m_{p}\left(r_{0}\right), \\
l_{p} \equiv l_{p}\left(r_{0}\right), & A \equiv A\left(r_{0}\right), \quad V_{p} \equiv V_{p}\left(r_{0}\right) .
\end{array}
$$

At $r_{0}$ there is a boundary that can be smooth or can have a shell. If there is a shell it can have zero or nonzero proper mass and zero or nonzero pressure.

For $r \geq r_{0}$ we assume that the solution is vacuum and thus that it is the Schwarzschild solution, $m(r)=$ $M$ constant, where $M$ is the spacetime mass or energy. In general $M$ and $m$ are different. Here we work with the case $M=m$ as we will see it is the case in the matching of the Zel'dovich-Letelier interior solution to the exterior Schwarzschild solution. Thus, $m$ is the mass of the spacetime.

There are two characteristic masses in this setting, the spacetime mass $m$ and the proper mass of the object $m_{p}$. It is then appropriate to define generically the mass defect $\Delta m$ of an object as

$$
\Delta m=m_{p}-m,
$$

which indicates how much mass, or energy, was put into the construction of the spacetime.

\section{B. Features of pit spacetimes with $m=0, r_{0}=0$, $\frac{2 m}{r_{0}}=1$, and maximal mass defect}

We put $M=m$, i.e., there is no contribution to the exterior spacetime mass from the boundary at $r_{0}$, and stick to calling it $m$. Considering the mass function $m(r)$ appearing in Eq. (11), we assume that $1-\frac{2 m(r)}{r}$ is uniformly bounded and write $1-\frac{2 m(r)}{r} \geq 0$, i.e.,

$$
\frac{2 m(r)}{r} \leq 1
$$

With this assumption we can make some general, concrete remarks. Define $\varepsilon$ as any positive number, which can be as small as we want, and $\chi(r)$ a function of $r$ always greater than zero, such that $1-\frac{2 m(r)}{r}=\varepsilon \chi(r)$. Take the maximal value of $\chi(r)$ as $\chi_{\max }$ and its minimum value as $\chi_{\min }$. Then, since Eq. (9) holds, the integral of Eq. (2) converges, and taking the integrals up to the boundary $r_{0}$ in Eqs. (2) and (3) leads to

$$
\frac{m}{\sqrt{\varepsilon \chi_{\max }}} \leq m_{p} \leq \frac{m}{\sqrt{\varepsilon \chi_{\min }}} .
$$

Take the quasiblack hole limit, i.e., $r_{0} \rightarrow 2 m$, or $\frac{2 m}{r_{0}} \rightarrow 1$ from below, so that one also has $\varepsilon \rightarrow 0$. This is a configuration made of some material with boundary radius at its own gravitational radius $2 m$, it is a configuration on the verge of becoming a black hole. Suppose that $m_{p}$ remains finite on this limit. Then, since $\varepsilon \rightarrow 0$, one has mandatorily from Eq. (10) that $m$ goes to zero, so, since $r_{0}=2 m$ in this limit, one also has $r_{0} \rightarrow 0$. Thus, one has an object that has $m=0$, i.e., zero mass energy $m$, and $r_{0}=0$, i.e., zero radius $r_{0}$, with $\frac{2 m}{r_{0}}=1$, and also has finite nonzero proper mass $m_{p}$. In addition, defining the mass defect $\Delta m=m_{p}-m$ as in Eq. (8), we see that this object has a maximal mass defect given by $\Delta m=m_{p}$. In brief, such an object has

$m=0, \quad r_{0}=0, \quad \frac{2 m}{r_{0}}=1, \quad m_{p}=$ finite,$\quad \Delta m=m_{p}$.

This is an amazing object. It has zero spacetime mass and zero area radius, and, although $m=0$, the ratio $\frac{2 m}{r_{0}}$ is not zero, but actually one. In addition, it has finite proper mass and maximal mass defect. We call this structure a pit, as it stores a nonzero proper mass in a zero spacetime mass spacetime with zero area radius. As $\frac{2 m}{r_{0}}=1$, the pit is indeed a quasiblack hole, although an atypical one. A specific realization of this general analysis is through the Zel'dovich-Letelier-Schwarzschild star that we will display next and where there are three possible classes, two of them being string pits, each with distinct and rather interesting features, and the other being a compact stringy star at the quasiblack hole state.

\section{THE \\ ZEL'DOVICH-LETELIER-SCHWARZSCHILD STAR AND ITS LIMITS}

\section{A. The interior, the shell junction, the exterior, and the Zel'dovich-Letelier-Schwarzschild star}

\section{The Zel'dovich-Letelier interior}

Let us be concrete. To simplify, let us choose an equation of state of the form $p_{r}=-\rho$. Then, inside for $r \leq r_{0}$, we have that indeed $\psi(r)=0$ and the only function that matters is the function $m(r)$ that appears in Eqs. (1) and (2). The conservation law $T_{; b}^{a b}=0$ with $a=r$ gives $p_{t}=p_{r}+\frac{r}{2} p_{r}^{\prime}$, and using the equation of state $p_{r}=-\rho$ one gets $p_{t}=-\rho-\frac{r}{2} \rho^{\prime}$. Following Zel'dovich [1] and Letelier [2], see also [3, 4], we put $\rho=\frac{b}{8 \pi r^{2}}$, where $b$ is a positive constant. Thus, the full general relativistic solution using the Einstein equation is

$$
\begin{gathered}
\rho=\frac{b}{8 \pi r^{2}}, \\
p_{r}=-\frac{b}{8 \pi r^{2}}, \\
p_{t}=0 .
\end{gathered}
$$

Since $p_{t}=0$, the source is string dust, strings in the radial direction up to $r_{0}$. Putting the energy-density expression Eq. (12) into Eq. (2), one obtains $m(r)=\frac{b}{2} r$, i.e., $\frac{2 m(r)}{r}=b$, and the line element, Eq. (1), becomes

$$
d s^{2}=-(1-b) d t^{2}+\frac{d r^{2}}{1-b}+r^{2} d \Omega^{2} .
$$


This metric yields a spacetime that has a spherical conic deficit. Indeed, redefining $\bar{t}=\sqrt{1-b} t$ and $\bar{r}=\frac{r}{\sqrt{1-b}}$, one gets the conical form of the metric, namely, $d s^{2}=$ $-d \bar{t}^{2}+d \bar{r}^{2}+\bar{r}^{2}(1-b) d \Omega^{2}$. Clearly, the inside metric is a deficit angle metric. Returning to the main functions of a static spherical symmetric spacetime, Eqs. (2)-(6), we can put them in the case of the Zel'dovich-Letelier spacetime in the form

$$
\begin{gathered}
m(r)=\frac{1}{2} b r \\
m_{p}(r)=\frac{b r}{2 \sqrt{1-b}}, \\
l_{p}(r)=\frac{r}{\sqrt{1-b}}, \\
A(r)=4 \pi r^{2}, \\
V_{p}(r)=\frac{4 \pi r^{3}}{3 \sqrt{1-b}} .
\end{gathered}
$$

Clearly, we can rewrite $m_{p}(r)$ as $m_{p}(r)=\frac{m(r)}{\sqrt{1-b}}, l_{p}(r)$ as $l_{p}(r)=\frac{2 m(r)}{b \sqrt{1-b}}=\frac{2 m_{p}(r)}{b}$, and $V_{p}(r)$ as $V_{p}(r)=\frac{32 m(r)^{3}}{3 b^{3} \sqrt{1-b}}=$ $\frac{32 m_{p}(r)^{3}(1-b)}{3 b^{3}}$. It is assumed that $b \leq 1$ so that the metric in Eq. (15) is static, and in addition it is assumed that the parameter $b$ is positive, so that the mass function $m(r)$ in Eq. (16) is positive, i.e., we put

$$
0<b \leq 1
$$

Equations. (15)-(20) with the condition (21) characterize the interior spacetime defined for $r \leq r_{0}$.

Note that the interior solution is singular at $r=$ 0 , the density and radial pressure, given in Eqs. (12) and (13), respectively, diverge there, and thus the Ricci and Riemann tensors and corresponding scalars diverge. Zel'dovich [1] deals with gravitational collapse issues, neglects $p_{r}$, and dismisses this singularity problem, showing that rounding up the energy density $\rho$ at the origin makes no difference for his final results. Letelier [2] suggests that the solution can be used as an intermediary solution between the Schwarzschild interior solution and a Schwarzschild exterior. Here we use the solution to match it to a Schwarzschild exterior, and in taking the limit $r_{0} \rightarrow 2 m$ it is found that this singularity is not naked because it is within a quasiblack hole.

\section{The shell junction}

The junction of the inside and the outside is at some $r_{0}$. At the junction $r_{0}$ we consider the metric to be of the form

$$
d s^{2}=-d \tau^{2}+r_{0}^{2} d \Omega^{2},
$$

where $\tau$ is the proper time at the junction. For the outside we consider a vacuum spacetime and thus from
Birkhoff's theorem it is the Schwarzschild spacetime. Since from the inside the radial pressure at $r_{0}$ is nonzero, namely, $p_{r}=-\frac{b}{4 \pi r_{0}^{2}}$, see Eq. (13), and from the outside $p_{r}=0$, as we consider that the outside is vacuum, there is a clear jump in the radial pressure that has to be smoothed out by a thin spherical shell at the junction at $r_{0}$. The energy-momentum tensor of the thin shell can be found. We write the contribution to the energy-momentum tensor $T^{a}{ }_{b}$ from the shell in the form $T^{a}{ }_{b}=S^{a}{ }_{b} \delta\left(l-l_{0}\right)$, where $S^{a}{ }_{b}$ is the intrinsic energy-momentum tensor associated with the shell, $\delta$ is the Dirac delta function, $l$ is the proper radial length in the neighborhood of the shell, and $l_{0}$ corresponds to the boundary at the shell. Then, following the junction formalism for general relativity [5], one finds $S^{\tau}{ }_{\tau}=0$ and $S^{\theta}{ }_{\theta}=S_{\phi}^{\phi}=\frac{b}{16 \pi r_{0} \sqrt{1-b}}$. Assuming that the shell is made of a perfect fluid and writing $S^{\tau}{ }_{\tau} \equiv-\sigma$ and $S_{\theta}^{\theta}=S_{\phi}^{\phi} \equiv P$, where $\sigma$ is the energy density of the shell and $P$ is the tangential pressure at the shell, we thus have

$$
\sigma=0, \quad P=\frac{b}{16 \pi r_{0} \sqrt{1-b}} .
$$

Note that there is no mass for the shell as $m_{\text {shell }}=$ $4 \pi r_{0}^{2} \sigma=0$. Note that $P$ closes the conical deficit set in by the interior spacetime such that the exterior Schwarzschild spacetime has no conical deficit. The shell's tangential pressure $P$ is there to close ends, literally.

\section{The exterior Schwarzschild}

The outer spacetime is vacuum, and therefore the exterior general relativistic metric, the metric for $r \geq r_{0}$, is Schwarzschild, i.e., $d s^{2}=-\left(1-\frac{2 M}{r}\right) d t^{2}+\frac{d r^{2}}{1-\frac{2 M}{r}}+r^{2} d \Omega^{2}$ for some spacetime mass $M$. In general $\stackrel{r}{M}$ and $m$ have different values. Here, since the shell has no mass, $m_{\text {shell }}=0$, we deal with the case $M=m$ and keep $m$ throughout. Thus, we put

$$
d s^{2}=-\left(1-\frac{2 m}{r}\right) d t^{2}+\frac{d r^{2}}{1-\frac{2 m}{r}}+r^{2} d \Omega^{2},
$$

as the exterior Schwarzschild metric.

\section{The full solution: The Zel'dovich-Letelier-Schwarzschild star}

The full general relativistic solution is composed of three parts. The inside with the metric given by Eq. (15), the shell with the metric given by Eq. (22), and the outside with the metric given in Eq. (24). The main global features can be found at the junction $r_{0}$. From Eqs. (16)(20) they are

$$
\begin{gathered}
m=\frac{1}{2} b r_{0}, \\
m_{p}=\frac{b r_{0}}{2 \sqrt{1-b}},
\end{gathered}
$$




$$
\begin{gathered}
l_{p}=\frac{r_{0}}{\sqrt{1-b}}, \\
A=4 \pi r_{0}^{2}, \\
V_{p}=\frac{4 \pi r_{0}^{3}}{3 \sqrt{1-b}} .
\end{gathered}
$$

We can rewrite $m_{p}$ as $m_{p}=\frac{m}{\sqrt{1-b}}, l_{p}$ as $l_{p}=\frac{2 m}{b \sqrt{1-b}}=$ $\frac{2 m_{p}}{b}$, and $V_{p}(r)$ as $V_{p}=\frac{32 m^{3}}{3 b^{3} \sqrt{1-b}}=\frac{32 m_{p}^{3}(1-b)}{3 b^{3}}$, and we recall that Eq. (21) should be taken into account, i.e., $0<b \leq 1$. This is the full Zel'dovich-LetelierSchwarzschild spacetime solution, i.e., the Zel'dovichLetelier-Schwarzschild star.

\section{B. The limit $r_{0} \rightarrow 2 m$ of the \\ Zel'dovich-Letelier-Schwarzschild star: Distinguishing features}

We are interested in the limit in which

$$
r_{0} \rightarrow 2 m,
$$

i.e., the quasiblack hole limit in which an object is at its own gravitational radius [6 10]. It follows from Eq. (25) that this means

$$
b \rightarrow 1
$$

Let us take $m_{p}$ in Eq. (26) as the quantity that identifies the possible different classes. To see this, we put $m_{p}$ as $m_{p}(1-b)^{\gamma}=\mu$ for some exponent $\gamma$ and some finite renormalized proper mass $\mu$, with $\mu \geq 0$. This choice for the relation between the proper mass $m_{p}$ and the renormalized mass $\mu$ is taken because it takes care of all the independent cases, actually three cases, when one takes the limit given in Eq. (31). For now we leave $b$ generic, only afterward do we take that limit. Then Eqs. (25)-(29) with $r_{0}=2 m$ of Eq. (30) give

$$
\begin{gathered}
m=\mu(1-b)^{\frac{1}{2}-\gamma}, \\
m_{p}=\mu(1-b)^{-\gamma}, \\
l_{p}=2 \mu \frac{(1-b)^{-\gamma}}{b}, \\
A=16 \pi \mu^{2} \frac{(1-b)^{1-2 \gamma}}{b^{2}}, \\
V_{p}=\frac{32}{3} \mu^{3} \frac{(1-b)^{1-3 \gamma}}{b^{3}},
\end{gathered}
$$

respectively. From Eqs. (32) and (33) we see that a negative exponent $\gamma$ gives zero mass $m$ and zero proper mass $m_{p}$ in the limit of Eq. (31). It is thus of no interest as it gives nothing, and we impose $\gamma \geq 0$. From Eq. (32) we see that an exponent $\gamma$ greater that $\frac{1}{2}$ gives an infinite mass $m$ in the limit of Eq. (31) and the spacetime with the line element given in Eq. (24) is not well defined, and we impose $\gamma \leq \frac{1}{2}$. Thus, $\gamma$ is within the range

$$
0 \leq \gamma \leq \frac{1}{2} .
$$

Then Eqs. (32) and (33) show that there are three distinct main classes: A. $\gamma=0$, which yields $m=0$ and $m_{p}$ equal to $\mu$, and thus $m_{p}$ is finite. It is a string pit solution; B. $0<\gamma<\frac{1}{2}$, which yields $m=0$ and $m_{p}$ infinite. It is also a string pit solution with different properties; C. $\gamma=\frac{1}{2}$, which yields $m$ finite and $m_{p}$ infinite. It is a compact stringy star with the highest compactification, it is not a pit. Let us analyze these three classes in detail.

\section{THE THREE LIMITING SOLUTIONS: TWO STRING PITS AND A COMPACT STRINGY STAR}

\section{A. A finite string in a pit, i.e., a string pit, almost detached from spacetime hanging from a point}

Here we find a finite string in a pit, a string pit, almost detached from spacetime hanging from a point, indeed with the spacetime mass $m=0$ and the proper mass $m_{p}=$ finite. This is the class $\gamma=0$.

We are interested in the limit in which $r_{0} \rightarrow 2 m$, see Eq. (30), i.e., the quasiblack hole state. It follows from Eq. (25) that this implies $b \rightarrow 1$, see Eq. (31). We put $\gamma=0$ in Eqs. (32)-(36) and analyze the spacetime's main features. From Eq. (32) we have $m \rightarrow 0$, i.e., $m=0$ in the limit. Equation (33) yields $m_{p}=\mu$, so $m_{p}$ is finite, with the subcase $m_{p}=0$ being a trivial case. From Eq. (34), the total proper length $l_{p}$ remains finite. This is clear as $l_{p}=2 m_{p}$ and $m_{p}$ is finite, see Eq. (27) with $b=1$. From Eq. (35), the surface area is $A=0$, and the area radius of the boundary is $r_{0}=0$. From Eq. (36), the proper volume is zero, $V_{p}=0$.

Thus, the full spacetime can be understood as follows. The inside solution is made of a one-dimensional open string, with finite length and zero volume. That the inside spacetime is a one-dimensional string can also be seen from the conical form of the inside metric, where for $b=1$ and $\bar{r}$ finite, as is the case, the angular part disappears leaving a one-dimensional space, i.e., a twodimensional spacetime. This single string in the inside spacetime pit is what is left from the hedgehog continuous spherical distribution of strings in the original Zel'dovichLetelier interior solution. It is a finite string almost detached from spacetime hanging from a point. For the shell, which joins the inside and the outside, one deduces it is now a point as $r_{0}=0$. Then, from Eq. (23), since $b=1$ and $r_{0}=0$, the tangential stresses tend to infinity, $P \rightarrow \infty$, and thus the point $r_{0}=0$ is singular, a type of singular horizon. For the outside, one has that the spacetime is Minkowski as $m=0$. Thus, in a nutshell, a Minkowski exterior spacetime hides a finite string pit. For a $t=$ constant and, e.g., $\theta=\frac{\pi}{2}$ space representation of the spacetime, see Fig. 1, where it is clear that the packed region with matter is a pit with a string hanging in the middle of flat space. 


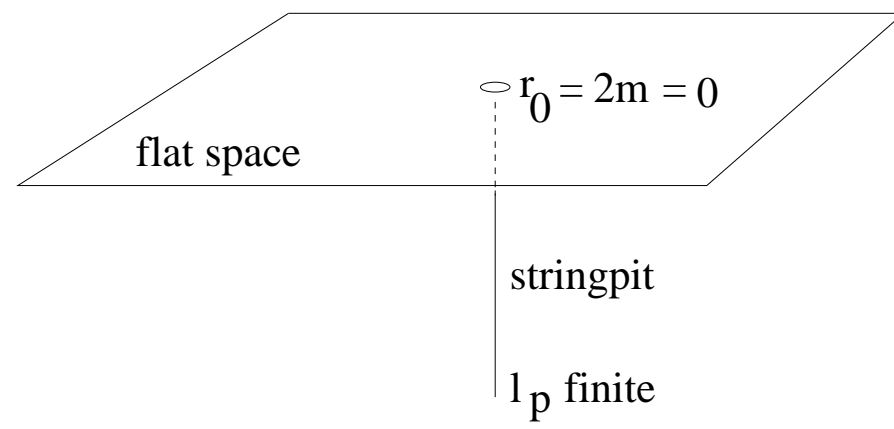

FIG. 1: A $t=$ constant and $\theta=\frac{\pi}{2}$ space representation of the spacetime given by the Zel'dovich-Letelier-Schwarzschild star with proper mass $m_{p}=\mu=$ finite; actually $m_{p}(1-b)^{\gamma}=\mu$, with $\gamma=0$ and $b=1$ and spacetime mass $m=0$. This class has $r_{0}=2 m$, and so it is a quasiblack hole, an atypical one, as it satisfies $r_{0}=2 m=0$. The space inside is a region of matter packed at the highest level, composed of a pit made of a one-dimensional string with finite proper length, hung from a point with $r_{0}=0$, which opens up to a massless $m=0$ Minkowski spacetime, i.e., a flat space here. The point $r_{0}=0$ yields the singular horizon of the quasiblack hole and joins the almost detached string to the rest of the space. Note that although $m=0$ and $r_{0}=0$ their ratio is finite as $\frac{2 m}{r_{0}}=1$. This object has maximal mass defect. The representation of this class of string pit solution shows clearly that the solution is a totally squashed Wheeler bag of gold.

Note five important and interesting properties of this class of string pit solution. First, the interior mass of the Zel'dovich-Letelier-Schwarzschild star [1-5] in this limit is hidden to the outside as it does not manifest itself gravitationally to the outer space since $m=0$. Second, it is also hidden because it is invisible since it is a quasiblack hole $6[-10]$. It is thus invisible for two reasons. Third, although $m=0$, its ratio to $r_{0}$ is finite, indeed, $\frac{2 m}{r_{0}}=1$. These three features characterize an atypical quasiblack hole. Thus, the dynamical gravitational collapse setting in [11 14] for which a null naked singularity, i.e., a singular horizon, forms when $m=0$ at $r=0$ and $\frac{2 m}{r}=1$ is also established in the static case that we are analyzing. Fourth, the mass defect, i.e., the proper mass minus the energy of the assembled object given in Eq. (8) is $\Delta m=m_{p}-m=m_{p}$, so we are in the presence of an object with maximal mass defect, see also [15, 16]. Fifth, it is a Wheeler bag of gold [17, 18] but is totally squashed.

For a study of the geodesics in this spacetime, see the Appendix A.

\section{B. A semi-infinite string in a pit, i.e., a string pit, almost detached from spacetime hanging from a point}

Here we find a semi-infinite string in a pit, a semiinfinite string pit, almost detached from spacetime hanging from a point, with indeed the spacetime mass $m=0$ and the proper mass $m_{p}=\infty$. This is the class $0<\gamma<$ $\frac{1}{2}$.

We are again interested in the limit in which $r_{0} \rightarrow 2 m$, see Eq. (30), i.e., the quasiblack hole state. It follows from Eq. (25) that again this implies that $b \rightarrow 1$, see Eq. (31). We put $0<\gamma<\frac{1}{2}$ in Eqs. (32)-(36) and analyze the main spacetime features. From Eq. (32) we have $m \rightarrow 0$, i.e., $m=0$ in the limit. Equation (33) yields $m_{p}=\infty$, the proper mass is infinite. From Eq. (34), the total proper length $l_{p}$ is then infinite. From Eq. (35) the surface area is $A=0$, and the area radius of the boundary is $r_{0}=0$. From Eq. (36), the proper volume for $0<\gamma<\frac{1}{3}$ is zero, $V_{p}=0$, for $\gamma=\frac{1}{3}$ it is finite nonzero, $V_{p}=\frac{32 \mu^{3}}{3}$, in which case it is a string or a rope with zero cross section area and infinite length but finite volume, and for $\frac{1}{3}<\gamma<\frac{1}{2}$ it is infinite, $V_{p}=\infty$.

Thus, the full spacetime can be understood as follows. The inside solution is made of a one-dimensional string, with infinite length, zero area, and zero, finite, or infinite volume depending on the specific $\gamma$. That the inside spacetime is a one-dimensional string can be also seen from the conical form of the inside metric, where for $b=1$ one has that $\bar{r}^{2}(1-b)$ tends to zero as is the case for the range of $\gamma$ under study, and thus the angular part disappears, leaving a one-dimensional space, i.e., a two-dimensional spacetime. This packed region of matter inside, made of a lonely boundless semi-infinite string in a pit almost detached from the outer spacetime hanging from a point, is the remnant of the infinite number of strings stemming radially from $r=0$ up to $r_{0}$ in a hedgehog distribution in the original Zel'dovich-Letelier interior solution. For the shell that joins the inside and the outside, one deduces it is now a point as $r_{0}=0$. Then, from Eq. (23), since $b=1$ and $r_{0}=0$, the tangential stresses tend to infinity, $P \rightarrow \infty$, and thus the point $r_{0}=0$ is singular, a type of singular horizon. For the outside, one has that the spacetime is Minkowski as $m=0$. Thus, in a nutshell, a Minkowski exterior space hides a semi-infinite string pit. For a $t=$ constant and, e.g., $\theta=\frac{\pi}{2}$ space representation of the spacetime, see Fig. 2 , where it is clear that the region packed with matter is a pit with a semi-infinite string hanging in the middle of flat space.

Note also five additional important and interesting properties of this class of string pit solutions. First, the interior mass of the Zel'dovich-Letelier-Schwarzschild star [1 5] in this limit is hidden to the outside, as it does not manifest itself gravitationally to the outer space since $m=0$. Second, it is also hidden because it is invisible since it is a quasiblack hole $[6-10]$. Thus, it is invisible for two reasons. Third, although $m=0$, its ratio to $r_{0}$ is finite,indeed, $\frac{2 m}{r_{0}}=1$, these three features characterizing an atypical quasiblack hole. Thus, the dynamical gravitational collapse setting in [11 14] for which a null naked singularity, i.e., a singular horizon, forms when $m=0$ at $r=0$ and $\frac{2 m}{r}=1$ is also established in the static case that we are analyzing. Fourth, the mass defect, i.e., the proper mass minus the energy of the assembled object given in Eq. (8) is $\Delta m=m_{p}-m=m_{p}=\infty$, so we are in the presence of an object with infinite mass defect, see also 15, 16. Fifth, it is a totally squeezed Wheeler bag of gold [17, 18] if we allow the bag to have infinite length.

The study of the geodesics in this spacetime can be done along the lines sketched in the previous spacetime. 


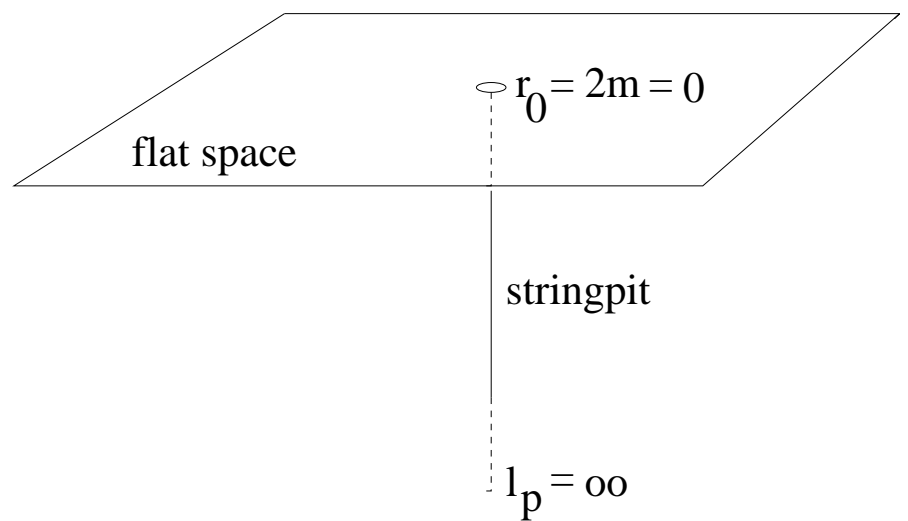

FIG. 2: A $t=$ constant and $\theta=\frac{\pi}{2}$ space representation of the spacetime given by the Zel'dovich-Letelier-Schwarzschild star with proper mass $m_{p}=$ infinite, actually $m_{p}(1-b)^{\gamma}=\mu$ with $0<\gamma<\frac{1}{2}$ and $b=1$ and spacetime mass $m=0$. This class has $r_{0}=2 m$, so it is a quasiblack hole, an atypical one, as it satisfies $r_{0}=2 m=0$. The space inside is a a region of matter packed at the highest level, composed of a pit made of a one-dimensional string with infinite proper length, hung from a point with zero area $A=0$ and $r_{0}=0$, and, depending on the parameter $\gamma$, with zero, finite, or infinite volume, hanged from a point with $r_{0}=0$, which opens up to a massless $m=0$ Minkowski spacetime, i.e., a flat space. The point $r_{0}=0$ yields the singular horizon of the quasiblack hole and joins the almost detached semi-infinite string to the rest of the space. Note that although $m=0$ and $r_{0}=0$ their ratio is finite, as $\frac{2 m}{r_{0}}=1$. This object has maximal mass defect, indeed infinite mass defect. The representation of the semiinfinite string pit solution shows that the solution is a totally squashed Wheeler bag of gold, although infinite in this class.

\section{A compact stringy star at its gravitational radius}

Here we find a compact stringy star at its gravitational radius, with spacetime mass $m=$ finite and the proper mass $m_{p}=\infty$. This is the class $\gamma=\frac{1}{2}$.

We are again interested in the limit in which $r_{0} \rightarrow 2 m$, see Eq. (30), i.e., the quasiblack hole state. It follows from Eq. (25) that again this implies that $b \rightarrow 1$, see Eq. (31). We put $\gamma=\frac{1}{2}$ into Eqs. (32)-(36) and analyze the main spacetime features. From Eq. (32) we have $m=\mu$, i.e., $m$ is finite in the limit. Equation (33) yields $m_{p}=\infty$, the proper mass is infinite. From Eq. (34), the total proper length $l_{p}$ is then infinite. From Eq. (35) the surface area $A=16 \pi \mathrm{m}^{2}$, which is finite, and the area radius of the boundary $r_{0}=2 m$ is also finite. From Eq. (36), the proper volume is infinite, $V_{p}=\infty$.

Thus, the full spacetime can be understood as follows. The inside solution is made of a bulk and all the strings from the original Zel'dovich-Letelier solution remain, but they are now hidden in a spacetime inside a horizon at finite nonzero area $A$ and finite $r_{0}$. Note that $\bar{r}_{0}$ is infinite in this case and the inside space is therefore three dimensional, not one dimensional as in the previous two classes. For the shell that joins the inside and the outside, one deduces it is a sphere with radius $r_{0}=2 \mathrm{~m}$. Then, from Eq. (23), since $b=1$, the tangential stresses tend to infinity, $P \rightarrow \infty$, and thus the horizon $r_{0}$ is a null naked horizon. For the outside, one has that the spacetime is Schwarzschild as $m$ is finite and not zero. In brief, the solution represents a compact stringy star at the quasiblack hole state, made of strings from $r=0$ to $r_{0}$, the compact star's boundary is a quasihorizon, and the outside is Schwarzschild. This solution is not a pit. For a $t=$ constant and, e.g., $\theta=\frac{\pi}{2}$ space representation of the spacetime see Fig. 3 , where it clear that the region packed with matter has finite boundary area radius $r_{0}$ and unbound volume.

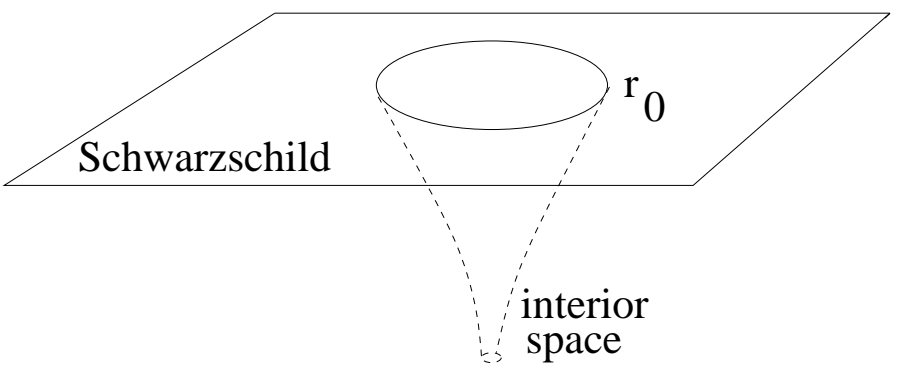

FIG. 3: A $t=$ constant and $\theta=\frac{\pi}{2}$ space representation of the spacetime given by the Zel'dovich-Letelier-Schwarzschild star with proper mass $m_{p}=\infty$, actually $m_{p}(1-b)^{\gamma}=\mu$, with $\gamma=\frac{1}{2}$ and $b=1$, and spacetime mass $m=$ finite. This class has $r_{0}=2 m$, so it is a quasiblack hole, a typical one, as it satisfies $r_{0}=2 m$ with $m$ finite. The space inside is an infinite volume region of matter, composed of the strings from the original Zel'dovich-Letelier solution but now hidden in a spacetime inside a horizon at finite nonzero $r_{0}$ that is singular and joins the inside to the curved Schwarzschild exterior space. Note that $\frac{2 m}{r_{0}}=1$, as it should be for a quasihorizon. This object has maximal, actually infinite, mass defect. The representation of the compact stringy star quasiblack hole solution shows that there is no Wheeler bag of gold in this class.

The five additional properties for this class of the compact stringy star solution can be put in the form. First, the interior mass of the Zel'dovich-Letelier-Schwarzschild star [1 [5] is not hidden in this class from the outside as it does manifest itself gravitationally to the outer space since $m$ is finite. Second, nonetheless it is still invisible since it is a quasiblack hole $[6[10]$. Third, here $\frac{2 m}{r_{0}}=1$ with $m$ and $r_{0}$ finite, so the solution is a quasiblack hole, a typical one in this class. In the dynamical gravitational collapse setting [11 14] there are also cases for which $\frac{2 m}{r}=1$, with $m$ finite, characterizing the formation of a typical black hole, not a naked singularity. Fourth, the mass defect, i.e., the proper mass minus the energy of the assembled object given in Eq. (8) is $\Delta m=m_{p}-m=m_{p}=\infty$, so we are in the presence of an object with infinite mass defect, see also [15, 16. Fifth, this class does not resemble a Wheeler bag of gold [17, 18] at all.

The study of the geodesics in this spacetime can be done along the lines sketched in the first spacetime. 


\section{CONCLUSIONS: SYNOPSIS, THERMODYNAMICS, AND CONNECTION TO OTHER WORKS}

\section{A. Synopsis of the three solutions}

The main results of this work are the finding of two classes of string pit solutions with unusual interesting structures and unusual interesting general relativistic gravitational fields. There is also another class, a compact stringy star solution that has standard properties. These three classes, although obtained from an appropriate limit of the Zel'dovich-Letelier-Schwarzschild star, stand on their own as separate general relativistic solutions, if one wishes to envisage them as such. In Table I, a summary of the main features of the three classes parametrized by the exponent $\gamma$ is displayed.

\begin{tabular}{|l|l|l|l|}
\hline Class & String $\mathrm{pit}_{1}$ & String $\mathrm{pit}_{2}$ & Compact stringy star \\
\hline$\gamma$ & 0 & $\left(0, \frac{1}{2}\right)$ & $\frac{1}{2}$ \\
\hline$m$ & 0 & 0 & Finite \\
\hline$m_{p}$ & Finite & Infinite & Infinite \\
\hline$l_{p}$ & Finite & Infinite & Infinite \\
\hline$A$ & 0 & 0 & Finite \\
\hline$V_{p}$ & 0 & 0, Finite, Infinite & Infinite \\
\hline
\end{tabular}

TABLE I: The main physical features along with its values of the three different classes of solutions, i.e., the first string pit class, the second string pit class, and the compact stringy star class, distinguished by the values of $\gamma$, namely, $\gamma=0$, $0<\gamma<\frac{1}{2}$, and $\gamma=\frac{1}{2}$, are displayed. The physical features are the spacetime mass $m$, the interior proper mass $m_{p}$, the interior proper length $l_{p}$, the surface area at the junction $A$, and the interior proper volume $V_{p}$. There is also the mass defect, i.e., the proper mass minus the spacetime mass or energy of the assembled object, $\Delta m=m_{p}-m$, which can be taken directly from the displayed values.

Some properties of the solutions in the three classes found here are as follows. (1) For the two first classes, comprising the string pit solutions that arise as the quasiblack hole limit of the Zel'dovich-Letelier-Schwarzschild star, in spite of having in the core a nonzero mass $m_{p}$, which in one class is arbitrarily large, this mass is hidden, as it does not manifest itself gravitationally to the outer spacetime since $m=0$. The third class, the compact stringy star solution, does not possess this property, the outer spacetime is Schwarzschild, and it has a finite nonzero $m$. (2) The three classes of solutions are invisible to the exterior since they are quasiblack holes, and as such no particle or light emanates from them. (3) The three class of solutions obey the quasiblack hole condition $\frac{2 m}{r_{0}}=1$. The two classes of string pit solutions are remarkable because they not only obey this condition, but have in addition $m=0$ and $r_{0}=0$, and they are indeed atypical quasiblack holes. These are the static solutions akin to the naked singularities, i.e., singular horizons, that form in dynamical gravitational collapse when $m=0$ at $r=0$, and $\frac{2 m}{r}=1$. The class of the compact stringy star solution has finite $m$ and finite $r_{0}$ and represents typical quasiblack holes, akin to the black holes that form in dynamical gravitational collapse with $m$ finite and some finite horizon radius $r$. Surprisingly, these classes of static solutions yield the same spectrum that appear in critical gravitational collapse. Indeed, in gravitational collapse there are solutions that yield naked null singularities that correspond here to the two string pit classes of solutions that also have naked null singularities, there are solutions that yield black holes that correspond here to the class of compact stringy stars at the quasiblack hole limit, and the solutions that disperse away in gravitational collapse here are the static Zel'dovichLetelier-Schwarzschild stars that we considered initially. (4) In the three classes, the mass defect, i.e., the proper mass $m_{p}$ minus the spacetime mass $m$ of the assembled objects, is maximal. In the first class of string pit solutions the mass defect is finite and maximal, and in the other two classes, i.e., the second class of string pit solutions and the compact stringy star solution, it is a superstrong mass defect, it is infinite. Moreover, as far as the gravitational mass defect is concerned, we have obtained general results, indeed we have shown that the maximal mass defect result can be obtained without specifying any equation of state. The Zel'dovich-Letelier-Schwarzschild star is a realization of the general result. (5) For the two first classes, i.e., the two string pit solutions, one finds Wheeler bags of gold, albeit totally squashed ones. The third class, the compact stringy star solution, has no bag. The finding of these three classes and the intepretation of them has benefited from several works [1 18].

\section{B. Thermodynamics of the three solutions}

It is also of interest to study the thermodynamic behavior of the string pits and stringy compact star quasiblack hole solutions which have an equation of state $p_{r}=-\rho$ for their interior. The appropriate thermodynamic formalism has been developed and is ready [19, 20]. When studying the thermodynamics of each system we suppose that a local temperature $T$ has been assigned to it. We deal with the main features of the temperature distribution and the entropy of the solutions, one at a time.

In relation to the temperature, in a gravitational system one has the Tolman temperature formula $T=$ $\frac{T_{0}}{\sqrt{1-\frac{2 m(r)}{r}}}$, for some local temperature $T$ of the system, which in general is different for each sphere with radius $r$, i.e., $T=T(r)$, and a temperature at infinity $T_{0}$, say, which has some constant value. Now, throughout the interior, for the string pit and the stringy star solutions one has that $\frac{2 m(r)}{r}$ is a constant, indeed, $\frac{2 m(r)}{r}=b$, and since $T_{0}$ is a constant, the whole interior solution, for any $r$, has the same local temperature $T(r)=\frac{T_{0}}{\sqrt{1-b}}$, a constant, so we can speak of an isothermal interior. Moreover, considering $T$ of the system finite. in the limit $b=1$ we then have $T_{0}=T \sqrt{1-b}=0$, so a remote observer will measure a vanishing temperature $T_{0}, T_{0}=0$, for these solutions. Usually, zero $T_{0}$ is a feature of an extreme 
quasiblack hole or of an extreme black hole. However, the systems we have analyzed are nonextremal. Thus, it seems that the string pits and the sringy star combine features of nonextremal and extremal quasiblack holes and black holes [19, 20].

In relation to the entropy, the entropy of a nonextremal quasiblack hole is $S=\frac{1}{4} A_{+}$, where $A_{+}=4 \pi r_{+}^{2}$ is the horizon area, with $r_{+}$being the horizon radius. Thus, it is the Bekenstein-Hawking formula for the entropy. Taking the limit $b=1, r_{0}$ is the radius of the system at the quasihorizon, $r_{0}=r_{+}$. For the string pits when $b=$ $1, r_{0}=r_{+}=0$, and thus $S=0$. Thus, entropically speaking, it has an extremal quasiblack hole behavior [20]. For the stringy star, $r_{0}=r_{+}$is finite and thus $S=\frac{1}{4} A_{+}$, which is a typical nonextremal quasiblack hole behavior.

\section{Connections of the three solutions to other works}

There are relevant and related solutions to the Zel'dovich-Letelier interior that have the mass function $m(r)$ obeying $\frac{2 m(r)}{r}=b$ but are not string dust, i.e., the equation of state is not $p_{r}=-\rho$. These interior related solutions when matching to a Schwarzschild exterior do not give the Zel'dovich-Letelier-Schwarzschild stars that we have been treating.

One example of such solutions is notable. In general relativity, using the Toman-Oppenheimer-Volkoff equation abbreviated usually to TOV equation, for the hydrostatic equilibrium of a spherically symmetric configuration with matter having an equation of state of the form $p=-q \rho$, with $p$ a perfect fluid pressure and $q$ some number, one finds that the energy density $\rho$ is proportional to $\frac{1}{r^{2}}$ and $m(r)$ indeed obeys $\frac{2 m(r)}{r}=b$, as reported with distinction in 21 23 and wrapped up and developed in [24, 25]. This general relativistic solution is called an isothermal perfect fluid solution because it is a generalization, albeit a nonisothermal one, of the Emden equation for isothermal spheres made of an ideal gas in Newtonian gravitation.

There are other instances where $\frac{2 m(r)}{r}=b$ appears. We mention regular black holes [26] where the solutions besides the $p_{r}=-\rho$ equation also have tangential pressure $p_{t}$ support, and general relativistic solutions coupled to nonlinear electrodynamics which have similar features [27]. We also allude to a thermodynamic treatment of the semiclassical degrees of freedom of a black hole which yields the expression $\frac{2 m(r)}{r}=b$ as a plausible equation [28].

One can only express wonder at all these interconnections from so many different settings.

\section{Acknowledgments}

JPSL thanks Fundação para a Ciência e Tecnologia - FCT, Portugal, for financial support through Grant No. UIDB/00099/2020. OBZ thanks Kazan Federal University for a state grant for scientific activities.
Appendix A: Geodesics in the spacetime of case A. A finite string in a pit, i.e., a string pit, almost detached from spacetime hanging from a point

\section{Timelike geodesics}

Here, we study radial timelike geodesics of the spacetime of case A. above, i.e., a finite string in a pit almost detached from spacetime hanging from a point, with $m=0$ and $m_{p}=$ finite. A radial geodesic has as one of its equations the equation $\left(1-\frac{2 m(r)}{r}\right) \dot{t}=E$, where the dot indicates derivative with respect to proper time $\tau$ and $E$ is a constant representing the energy per unit mass of the massive test particle along the geodesic. The other equation is $\left(1-\frac{2 m(r)}{r}\right) \dot{t}^{2}-\frac{\dot{r}^{2}}{1-\frac{2 m(r)}{r}}=1$. Combining the two, one gets $\dot{r}^{2}=E^{2}-1+\frac{2 m(r)}{r}$. Thus, $d \tau= \pm \frac{d r}{\sqrt{E^{2}-1+\frac{2 m(r)}{r}}}$. For the inside $\frac{2 m(r)}{r}=b$, so letting a massive test particle fall from some $r$ in the inside region to the center gives the proper time $\tau=\int_{0}^{r} \frac{d r^{\prime}}{\sqrt{E^{2}-1+b}}$, which yields $\tau=\frac{r}{\sqrt{E^{2}-1+b}}$. If the particle comes from $r_{0}$ with $E \geq 1$, then

$$
\tau_{\text {in }}=\frac{r_{0}}{\sqrt{E^{2}-1+b}} .
$$

In the limiting spacetime, $b \rightarrow 1$ and $r_{0} \rightarrow 0$ yields $\tau_{\text {in }}=$ 0 .

\section{Null geodesics}

Here, we study radial null geodesics of the spacetime of case A. above, i.e., a finite string in a pit almost detached from spacetime hanging from a point, with $m=0$ and $m_{p}=$ finite. For a null geodesic $d s^{2}=0$. Thus, the time between the center and some $r$ is $t=\int_{0}^{r} \frac{d r^{\prime}}{1-\frac{2 m\left(r^{\prime}\right)}{r^{\prime}}}$. For the inside $\frac{2 m(r)}{r}=b$, so the time between the center and the boundary $r_{0}$ is

$$
t_{\mathrm{in}}=\frac{r_{0}}{1-b} .
$$

In the limiting spacetime, $b \rightarrow 1$ and $r_{0} \rightarrow 0$, and taking into account Eqs. (16) and (17) with $m_{p}$ finite, one has $t_{\text {in }} \rightarrow \infty$ with $\frac{1}{\sqrt{1-b}}$. The time between $r_{0}$ and some $r_{1}>r_{0}$ is $t_{\text {out }}=\int_{r_{0}}^{r_{1}} \frac{d r}{1-\frac{2 m\left(r_{0}\right)}{r}}=\int_{r_{0}}^{r_{1}} \frac{d r}{1-\frac{b r_{0}}{r}}=\int_{r_{0}}^{r_{1}} \frac{d r r}{r-b r_{0}}=$ $r_{1}-r_{0}+b r_{0} \ln \frac{r_{1}-b r_{0}}{r_{0}(1-b)}$, i.e.,

$$
t_{\text {out }}=r_{1}-r_{0}+b r_{0} \ln \frac{r_{1}-b r_{0}}{r_{0}-b r_{0}}
$$

When $b \rightarrow 1, t_{\text {out }} \rightarrow \infty$. The divergences of $t_{\text {in }}$ are stronger than those of $t_{\text {out }}$.

\section{Redshift and blueshift of light}

Now we analyze the redshift and blueshift of light of the spacetime of case A. above, i.e., a finite string in a pit 
almost detached from spacetime hanging from a point, with $m=0$ and $m_{p}=$ finite. According to the standard formulas,

$$
\omega=\frac{\omega_{0}}{\sqrt{1-\frac{2 m(r)}{r}}},
$$

where $\omega$ is the frequency measured by a local static observer at a given point $r$ and $\omega_{0}$ is a constant. Inside, one has $1-\frac{2 m(r)}{r}=1-b$, and outside $1-\frac{2 m(r)}{r}=1-\frac{2 m}{r}$. Thus, inside, for $r \leq r_{0}$, the frequency of light does not change as it propagates. On the other outside, $r \geq r_{0}$, one can put $\omega_{0} \equiv \omega_{\infty}$, the frequency at infinity. Therefore, when light goes from $r_{0}$ to an even larger $r$, it will arrive at infinity with $\omega_{\infty}=\omega \sqrt{1-b}$. In the limit $b \rightarrow 1$, we have an infinite redshift, as expected for a quasiblack hole. If light with finite $\omega_{\infty}$ comes from infinity and enters the inner region, it has $\omega=\frac{\omega_{\infty}}{\sqrt{1-b}}$ there.
[1] Ya. B. Zel'dovich, "The collapse of a small mass in the general theory of relativity", Zh. Eksp. Teor. Fiz. 42, 641 (1962); English translation, Sov. Phys. JETP 15, 446 (1962).

[2] P. S. Letelier, "Clouds of strings in general relativity", Phys. Rev. D 20, 1294 (1979).

[3] M. Barriola and A. Vilenkin, "Gravitational field of a global monopole", Phys. Rev. Lett. 63, 341 (1989).

[4] E. I. Guendelman and A. Rabinowitz, "Gravitational field of a hedgehog and the evolution of vacuum bubbles", Phys. Rev. D 44, 3152 (1991).

[5] W. Israel, "Singular hypersurfaces and thin shells in general relativity", Nuovo Cimento B 44, 1 (1966).

[6] J. P. S. Lemos and O. B. Zaslavskii, "Quasiblack holes: Definition and general properties", Phys. Rev. D 76, 084030 (2007); arXiv:0707.1094 [gr-qc].

[7] J. P. S. Lemos and O. B. Zaslavskii, "Black hole mimickers: Regular versus singular behavior", Phys. Rev. D 78, 024040 (2008); arXiv:0806.0845 [gr-qc].

[8] J. P. S. Lemos and O. B. Zaslavskii, "Quasiblack holes with pressure: General exact results", Phys. Rev. D 82, 024029 (2010); arXiv:1004.4651 [gr-qc].

[9] J. P. S. Lemos and O. B. Zaslavskii, "Quasiblack holes: Properties and Carter-Penrose diagrams", in Proceedings of the 13th Marcel Grossmann Meeting (Stockholm, 2012), edited by K. Rosquist et al (World Scientific, Singapore, 2015), p. 1195.

[10] J. P. S. Lemos and O. B. Zaslavskii, "Compact objects in general relativity: From Buchdahl stars to quasiblack holes", Int. J. Mod. Phys. D 29, 2041019 (2020); arXiv:2007.00665 [gr-qc].

[11] D. Lynden-Bell and J. P. S. Lemos, "On Penston's solutions for cold collapse", Mon. Not. R. Astron. Soc. 233, 197 (1988).

[12] J. P. S. Lemos and D. Lynden-Bell, "A general class of spherical Newtonian self-similar solutions for a cold fluid II: Solutions with gravity", Mon. Not. R. Astron. Soc. 240, 317 (1989).

[13] J. P. S. Lemos, "Naked singularities: Gravitationally collapsing configurations of dust or radiation in spherical symmetry - a unified treatment", Phys. Rev. Lett. 68, 1447 (1992).

[14] M. W. Choptuik, "Universality and scaling in gravitational collapse of a massless scalar field", Phys. Rev. Lett. 70, 9 (1993).

[15] V. A. Ruban, "Spherically symmetric T-models in the general theory of relativity", Zh. Eksp. Teor. Fiz. 56, 1914 (1969); English translation, Sov. Phys. JETP 29, 1027 (1969) and Gen. Relativ. Gravit. 33, 375 (2001).

[16] O. B. Zaslavskii, "Ultimate gravitational mass defect", Gen. Relativ. Gravit. 38, 945 (2006); arXiv:gr-qc/0511063

[17] J. A. Wheeler, "Geometrodynamics and the issue of the final state", in Relativity, Groups, and Topology, edited by C. DeWitt and B. DeWitt (Gordon and Breach, New York, 1964), p. 315.

[18] Y. C. Ong, "The persistence of the large volumes in black holes", Gen. Relativ. Gravit. 47, 88 (2015); arXiv:1503.08245 [gr-qc].

[19] J. P. S. Lemos and O. B. Zaslavskii, "Entropy of quasiblack holes", Phys. Rev. D 81, 064012 (2010); arXiv:0904.1741 [gr-qc].

[20] J. P. S. Lemos and O. B. Zaslavskii, "Entropy of extremal black holes from entropy of quasiblack holes", Phys. Lett. B 695, 37 (2011); arXiv:1011.2768 [gr-qc].

[21] O. Klein, "On a case of radiation equilibrium in general relativity theory and its bearing on the early stage of stellar evolution", Ark. Mat. Astron.Fys. A (Stockholm) 34A, 1 (1947).

[22] C. W. Misner and H. Zapolsky, "High density behavior and dynamical stability of neutron star models", Phys. Rev. Lett. 12, 635 (1964).

[23] S. Chandrasekhar, "A limiting case of relativistic equilibrium", in General Relativity: Papers in Honour of J. L. Synge, edited by L. O'Raifeartaigh (Clarendon Press, Oxford, 1972), p. 185.

[24] P.-H. Chavanis, "Relativistic stars with a linear equation of state: Analogy with classical isothermal spheres and black holes", Astron. Astrophys. 483, 673 (2008), arXiv:0707.2292 [astro-ph].

[25] P. Beltracchi and P. Gondolo, "A curious general relativistic sphere", arXiv:1910.08166 [gr-qc] (2019).

[26] I. Dymnikova, "Vacuum nonsingular black hole", Gen. Relativ. Gravit. 24, 235 (1992).

[27] O. B. Zaslavskii, "Zel'dovich states with very small mass and charge in nonlinear electrodynamics coupled to gravity", Gravitation Cosmol. 16, 168 (2010); arXiv:1003.2324 [gr-qc].

[28] V. Berezin, "Black hole thermodynamics without a black hole?", Nucl. Phys. B 661, 409 (2003); arXiv:gr-qc/0302066 